\title{
Noninvasive Ventilation and Mechanical Insufflator-Exsufflator for Acute Respiratory Failure in Children With Neuromuscular Disorders
}

\author{
Tai-Heng Chen ${ }^{1,2,3}$ and Jong-Hau Hsu ${ }^{1,4 *}$ \\ 1 Department of Pediatrics, Kaohsiung Medical University Hospital, Kaohsiung Medical University, Kaohsiung, Taiwan, \\ ${ }^{2}$ Department of Emergency, College of Medicine, Kaohsiung Medical University, Kaohsiung, Taiwan, ${ }^{3}$ Department of \\ Biological Sciences, University of Southern California, Los Angeles, CA, United States, ${ }^{4}$ Department of Pediatrics, College of \\ Medicine, Kaohsiung Medical University, Kaohsiung, Taiwan
}

OPEN ACCESS

Edited by:

Ron Rubenstein,

Washington University in St. Louis,

United States

Reviewed by:

Oren Kupfer

Children's Hospital Colorado,

United States

Ignacio Tapia

Children's Hospital of Philadelphia

United States

*Correspondence:

Jong-Hau Hsu

jhh936@yahoo.com.tw

Specialty section:

This article was submitted to

Pediatric Pulmonology,

a section of the journal

Frontiers in Pediatrics

Received: 10 August 2020 Accepted: 12 October 2020

Published: 30 October 2020

Citation:

Chen T-H and Hsu J-H (2020)

Noninvasive Ventilation and Mechanical Insufflator-Exsufflator for Acute Respiratory Failure in Children

With Neuromuscular Disorders.

Front. Pediatr. 8:593282.

doi: $10.3389 /$ fped.2020.593282
Children with neuromuscular disorder (NMD) usually have pulmonary involvement characterized by weakened respiratory muscles, insufficient coughing, and inability to clear airway secretions. When suffering from community-acquired pneumonia, these patients are more likely to develop acute respiratory failure (ARF). Therefore, recurrent pneumonias leading to acute on chronic respiratory failure accounts for a common cause of mortality in children with NMD. For many years, noninvasive ventilation (NIV) has been regarded as a life-prolonging tool and has been used as the preferred intervention for treating chronic hypoventilation in patients with advanced NMD. However, an increasing number of studies have proposed the utility of NIV as first-line management for acute on chronic respiratory failure in NMD patients. The benefits of NIV support in acute settings include avoiding invasive mechanical ventilation, shorter intensive care unit or hospital stays, facilitation of extubation, and improved overall survival. As the difficulty in clearing respiratory secretions is considered a significant risk factor attributing to NIV failure, combined coughing assistance of mechanical insufflator-exsufflator (MI-E) with NIV has been recommended the treatment of acute neuromuscular respiratory failure. Several recent studies have demonstrated the feasibility and effectiveness of combined NIV and MI-E in treating ARF of children with NMD in acute care settings. However, to date, only one randomized controlled study has investigated the efficacy of NIV in childhood ARF, but subjects with underlying NMD were excluded. It reflects the need for more studies to elaborate evidence-based practice, especially the combined NIV and MI-E use in children with acute neuromuscular respiratory failure. In this article, we will review the feasibility, effectiveness, predictors of outcome, and perspectives of novel applications of combined NIV and MI-E in the treatment of ARF in NMD children.

Keywords: noninvasive ventilation, neuromuscular disorder, acute respiratory failure, mechanically assisted coughing, risk factors 


\section{PATHOPHYSIOLOGY UNDERLYING ACUTE RESPIRATORY FAILURE IN CHILDREN WITH NEUROMUSCULAR DISORDER}

Neuromuscular disease (NMD) is a heterogeneous group of diseases caused by various defects from multiple sources, including skeletal muscle, motor neurons, peripheral nerves, and neuromuscular junctions (1-4). Most primary NMD is associated with an inherited gene defect and usually onset in childhood with progressive degeneration. Due to weakened either one or all of the main respiratory muscle groups and impaired coughing ability, the respiratory dysfunction represents not only a critical health issue but a frequent unmet medical need of NMD patients $(2,5,6)$.

Children with NMD may have progressively developed chronic respiratory failure in the process of disease course.
However, episodic attacks of acute respiratory failure (ARF) may further aggravate the already existed respiratory compromises (7). Factors posing a risk of ARF in children with NMD are usually multifactorial and occur simultaneously (8-11). Table $\mathbf{1}$ summarizes the risk levels of various NMD potentially affected by the acute respiratory compromise. According to the timing of ARF occurrence, NMD can also be classified into two main categories: (1) early-onset (may as early as in neonatal period) with rapidly progressive NMD with acute episodes of respiratory failure; (2) late-onset and slowly progressive NMD with acute exacerbations of chronic respiratory failure $(12,14)$.

As shown in Figure 1, the pathophysiological mechanism of respiratory muscle groups involved in NMD patients can be summarized into three main components and several predisposing factors $(6,7,9,13,14)$. First, the weakness of bulbar muscles impedes the protection against the risk of aspiration

TABLE 1 | Risk levels and susceptible age groups of acute respiratory compromises in different neuromuscular disorders.

\begin{tabular}{|c|c|c|}
\hline Primarily affected age group & Risk level of ARF occurrence & Affected NMD \\
\hline Infant-to-adult life & Very high risk & $\begin{array}{l}\text { Some limb-girdle muscular dystrophy (LGMD), especially with sarcoglycanopathies (LGMD } \\
\text { types 2C, 2D, 2E, 2F) and LGMD type } 2 \text { I } \\
\text { Some CMD, especially merosin negative types 1A, 1B, 1C } \\
\text { Some myofibrillar myopathies (e.g., hereditary myopathy with early respiratory failure) } \\
\text { Early-onset infantile facioscapulohumeral muscular dystrophy (FSHD) } \\
\text { Early-onset Charcote-Marie-Tooth disease (CMTD) especially with GDAP1 mutation } \\
\text { Some congenital myopathies (e.g., severe recessive type of central core myopathy) }\end{array}$ \\
\hline & Intermediate risk & $\begin{array}{l}\text { Becker muscular dystrophy (BMD) } \\
\text { SMA type } 3 \\
\text { Inflammatory myopathies (e.g., polymyositis, dermatomyositis) } \\
\text { Classical type of FSHD } \\
\text { Some types of Charcot-Marie-Tooth disease (e.g., CMTD type 1B and 4) } \\
\text { Some congenital myopathies } \\
\text { Some mitochondrial myopathies } \\
\text { Guillain-Barré syndrome (GBS) } \\
\text { Myasthenia gravis (MG) }\end{array}$ \\
\hline
\end{tabular}

*Novel therapies are currently available (e.g., enzyme replacement, antisense nucleotide, and gene therapy) to be delivered in the neonatal period. Data of this table are modified and summarized from references: (11-13). 


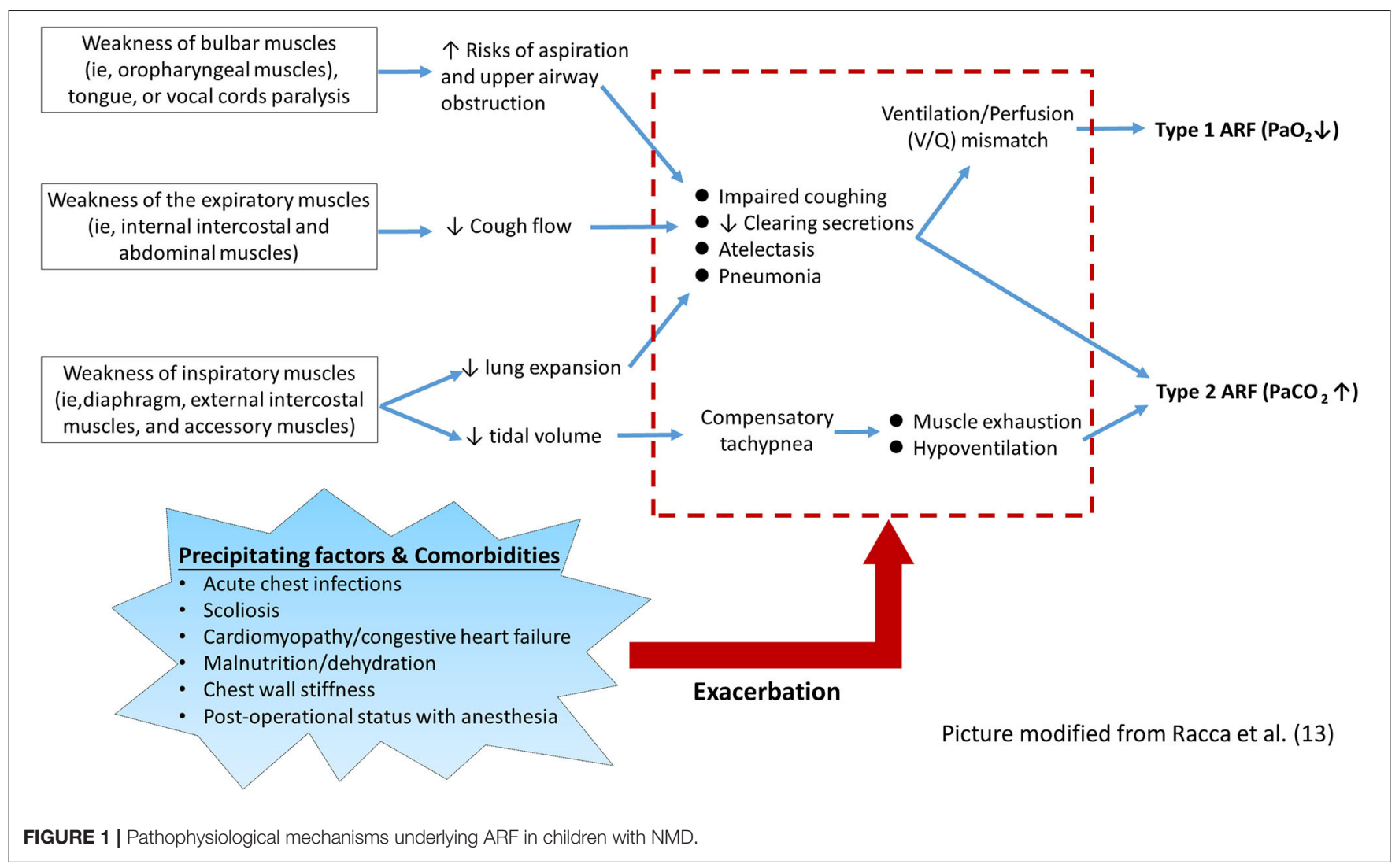

of the food or airway secretions, which may lead to frequent atelectasis and pneumonia (14). Additionally, weakness of bulbar muscles and tongue, and paralysis of vocal cords may cause mechanical obstruction of the upper airway, particularly in the supine position, and increase the likelihood of aspiration $(9,14)$. Second, weakness of the inspiratory muscles leads to reduced lung expansion and impaired coughing ability, which may lead to a ventilation/perfusion mismatch and consequent hypoxemia. Compensatory tachypnea due to small tidal volumes may further increase the mechanical load on already weakened respiratory muscles $(6,7,13)$. Third, the weakness of expiratory muscles leads to ineffective coughing and encumbrance of airway secretion, which consequently increases breathing load (14).

On the other hand, other systemic involvements associated with NMD may further aggravate the impairment of lung function, which precipitate the occurrence of $\operatorname{ARF}(5,6,14$, 15). In the advanced stage of NMD, progressive scoliosis is common and usually causes reduced chest wall compliance and unequal lung expansion. Patients with certain types of NMD, such as Duchenne muscular dystrophy and Emery-Dreifuss muscular dystrophy, frequently have cardiac involvement that may further worsen the respiratory function (e.g., pulmonary edema related to congestive heart failure) $(11,12)$. ARF may also occur in the perioperative period of some major surgeries, for example, correction of scoliosis or insertion of percutaneous gastrostomy. Such ARF episodes usually happen after extubation and are associated with bulbar dysfunction, postoperative pain, use of pain medications, or atelectasis caused by mucus plugging $(16,17)$. Malnutrition and dehydration developing during an acute illness should be aggressively intervened, as unmet caloric and metabolic needs may further aggravate ARF. Thus, each of these comorbidities necessitates multidisciplinary interventions and meticulous monitoring (5, 18-23).

In most cases, the occurrence of ARF in children with NMD is usually initiated by an upper respiratory tract infection, followed by complications of congested airway secretions, mucus plugs, and atelectasis $(9,24,25)$. In addition, increased nasal airflow resistance with nasal congestion in the setting of pre-existing upper airway obstruction from bulbar dysfunction also increases respiratory muscle load in the absence of bronchial secretions. Due to community pneumonia, the decreased lung compliance and the increased workload of already weak muscles may further contribute to the onset of ARF (6). Among children with $\mathrm{NMD}, \mathrm{ARF}$ is the main cause of unscheduled admissions and prolonged stay in the pediatric intensive care unit (ICU) $(11,26)$. Moreover, complications known to be associated with prolonged ICU stay and conventional invasive mechanical ventilator (IMV) may also contribute to high ICU mortality $(27,28)$. As a consequence, acute-on-chronic respiratory failure represents the most common cause of morbidity and mortality in children with $\operatorname{NMD}(9,29)$. 

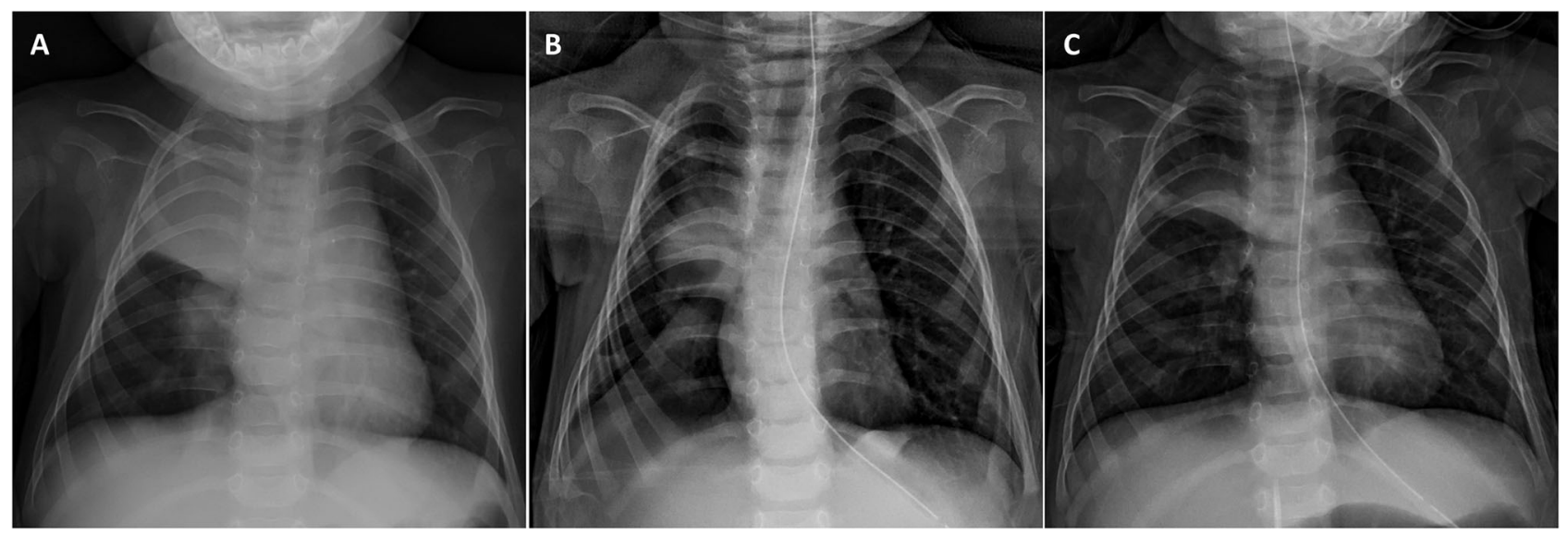

FIGURE 2 | Resolution of right upper lobe opacification in an infant with severe type 1 spinal muscular atrophy (SMA) after combining NIV and MI-E. (A) Chest X-ray on admission showing right lung pneumonia with significant atelectasis complicated by copious secretions. (B) A significant improvement was found after 2 -days treatment, with a resolution of atelectasis. (C) A progressive improvement of the pneumonic patch was observed on day 7 when discharged from PICU.

\section{NONINVASIVE VENTILATION IN CHILDHOOD ACUTE NEUROMUSCULAR RESPIRATORY FAILURE}

In the past few decades, noninvasive ventilation (NIV) has been regarded as a life-prolonging tool for managing chronic respiratory failure in patients with $\operatorname{NMD}(6,11,21,30)$. On the other hand, recent studies and guidelines have also proposed the role of NIV as a first-line intervention for ARF in NMD patients to avoid endotracheal intubation and the use of invasive mechanical ventilation $(\operatorname{IMV})(11,31,32)$. Support for alternative use of NIV is based on concerns about the many complications of IMV use in patients with NMD. These include laryngeal edema, subglottic stenosis, barotrauma, and ventilator-associated pneumonia, leading to subsequent tracheotomy and poor quality of life (17, 33-35). Besides, long-term dependence on IMV and prolonged ICU stay are associated with nosocomial infections, aspiration, atelectasis, thromboembolic events, contractures, and bedsores, all of which can lead to high mortality in NMD patients (8). In this regard, emerging evidence supports the alternative NIV administration to manage ARF in patients with NMD (36-38). Indeed, several studies have indicated several potential benefits of NIV in treating ARF of NMD patients, including shortening the ICU and hospital stay, facilitating extubation, and improving the overall survival (16, 39-42).

\section{ROLE OF AGGRESSIVE SECRETION MANAGEMENT IN MANAGING ARF OF NMD CHILDREN}

Mucociliary clearance is generally not affected by NMD, except for damage to the ciliary epithelium due to repeat aspiration or acute chest infection (43). Aggressive secretion clearance is crucial for children with NMD to avoid progression to severe respiratory compromises during respiratory infections $(44,45)$.
Also, excessive secretion has been regarded as a major risk factor causing NIV failure in treating ARF of NMD patients $(6,46,47)$. Therefore, facilitating secretion clearance and normalizing gas exchange by augmenting cough ability is the mainstay to treat ARF in children with NMD (48).

Although NMD patients rarely achieve sufficient chest and abdomen pressure due to the weakness of the intercostal and abdominal muscles, the coughing can be augmented manually or mechanically. Among various coughing-assist techniques, the mechanical insufflator-exsufflator (MI-E) represents the most powerful tool that can promote the most effective peak flow to expel mucus plugging and resolve atelectasis (7, 49, 50). MIE can deliver a brief positive inspiratory pressure through a mask, mouthpiece, tracheostomy, or endotracheal tube to fully expand the chest, allowing air to enter the distal end of the mucus plugging, and then applying negative pressure, resulting in expiratory "cough" flow to remove airway secretions (45). A previous study showed that MI-E is superior to manual cough assistance in increasing cough flow in healthy subjects as well as in patients with amyotrophic lateral sclerosis (ALS), regardless of bulbar weakness (51). The additional use of MI-E helps to resolve excessive secretions and eliminate the risk of NIV failure in treating ARF of NMD patients. Therefore, recent evidence suggests that combining NIV and MI-E can be used as the firstline treatment for ARF in children with $\operatorname{NMD}(14,44,45,47,50)$. Our experiences also show that it can effectively treat ARF even for the most severe types of NMD (Figure 2).

\section{EFFECTIVENESS OF NIV IN POST-EXTUBATION SUPPORT FOR CHILDREN WITH NMD}

After recovering from an acute illness or surgery requiring sedation, a considerable number of NMD patients may not pass the IMV-dependent weaning tests, resulting in a high failure rate 


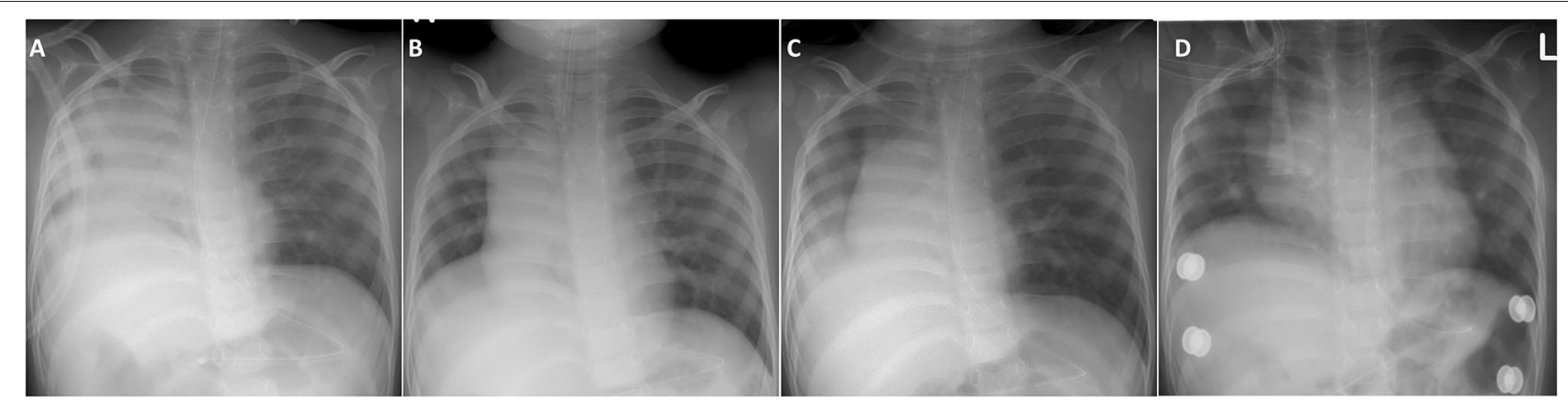

FIGURE 3 | Demonstration of chest X-ray in a toddler with congenital myopathy who immediately received NIV and MI-E for post-extubation respiratory support. (A) Previously failed extubation in another hospital was related to frequent right lung atelectasis and mucus plugging developing soon after extubation. (B) In our hospital, appropriate expansion of both lungs were noted before extubation. (C) Day 2 post-extubation showed mild right lung infiltration without atelectasis. (D) Discharge from PICU on day 7 post-extubation showed re-expansion of both lungs.

of extubation (26-28). Post-extubation ARF in NMD patients shares several pathomechanism features with episodic ARF, such as weak respiration drive, airway mucus-plugging due to difficulty in expectorating secretion, mostly categorized as type 2 (hypercapnic) ARF (28). The advent of active NIV support reduces the need for extensive weaning trials before extubation, which requires prolonged pressure support and spontaneous breathing. Some studies have validated that prompt NIV and MI-E use after extubation can significantly eliminate the risk of reintubation in NMD patients $(40,52,53)$. There is a general agreement that, if not contraindicated (e.g., uncontrolled airway secretions or severe bulbar dysfunction), patients with chronic NMD should be extubated directly to NIV combined with MIE $(28,54)$. The effectiveness of this NIV support is significant in preventing reintubation in young children with NMD (Figure 3).

\section{REVIEW OF CLINICAL STUDIES ON NIV FOR THE TREATMENT OF ACUTE-ON-CHRONIC NEUROMUSCULAR RESPIRATORY FAILURE}

There are relatively few prospective studies on the management of NMD patients with ARF, which may be because most chronic NMDs are rare diseases, making it difficult to recruit patients. As shown in Table 2, evidence that NIV can help avoid intubation of patients with chronic NMD during the ARF episodes comes from 11 non-randomized observational studies of a total of 178 subjects (age range 2 months to 69 years), of which most subjects are known to be under 25 years $(36,37,39-42,52,53,56,57)$. However, in most studies, there are few descriptions of methods to manage airway secretions, and its role in contributing to the success of NIV in treating ARF is not well defined (45). Even though an increasing number of studies have recognized the benefit of combined NIV and MI-E use in the ARF management and facilitation of extubation in adult NMD patients $(37,40,54)$, similar studies on the pediatric NMD populations are scarce. In heterogeneous pediatric populations, several risk factors for predicting the failure of NIV treatment fo ARF have been reported $(11,32,44)$, but it is still unclear whether similar factors exist in a specific pediatric NMD population.

However, only one randomized controlled study has investigated the efficacy of NIV in treating children with ARF but has excluded children with underlying NMD (31). Two recent studies reported by the same team described the protocol and effectiveness of a combination of NIV with MI-E in treating ARF of children with chronic NMD $(52,57)$. The pilot study of children encompassing various NMDs has demonstrated the feasibility of this combined noninvasive approach. The following research on a larger cohort of NMD patients further verified its safety and effectiveness. Overall, combining the data of these two studies on 71 NMD patients shows that timely implementation of NIV and MI-E can avoid intubation or reintubation in $75-86 \%$ of ARF events, of which $80 \%$ are pediatric cases. The PICU and hospital stay of children successfully rescued through NIV/MI-E is shorter than that of children who received intubation. Besides, several predictors of NIV failure were identified, including physical parameters (changes in respiratory rate) and laboratory variables (changes in $\mathrm{PaCO}_{2}$ and $\mathrm{pH}$ value of arterial blood gas).

\section{COMBINED NIV AND MI-E IN ARF TREATMENT OF NMD CHILDREN}

The interface connects the ventilator tubing to the patient to deliver pressurized gas to the airway during NIV administration. It may take several attempts to find a suitable interface, but this is the key to successfully treating ARF in NMD children with NIV while minimizing air leakage, maximizing patient comfort, and synchronizing with the ventilator $(44,58,59)$. However, although interface tolerance is a pivotal factor associated with NIV success, comparative data on the interface of infants and young children is scarce (60).

A transparent interface is highly recommended to ensure correct positioning and enhance patient monitoring (59). The medical team should be well trained to select the most suitable interface individualized for each critically ill child (61). As proof of principle, the smallest interface with the least air leakage should be selected to minimize the dead space. For infants, 
TABLE 2 | Noninvasive airway approaches for patients with NMD with acute on chronic respiratory failure.

\begin{tabular}{|c|c|c|c|c|c|c|c|c|c|}
\hline References & $\begin{array}{l}\text { Study } \\
\text { Design }\end{array}$ & $\begin{array}{l}\text { Number of NMD } \\
\text { patients (age) }\end{array}$ & $\begin{array}{l}\text { NMD diagnosis } \\
\text { (n) }\end{array}$ & ARF types* $(n, \%)$ & $\begin{array}{l}\text { NIV/interface/ } \\
\text { secretion } \\
\text { clearance }\end{array}$ & Success rate and main findings & $\begin{array}{l}\text { Predictor of } \\
\text { NIV failure }\end{array}$ & $\begin{array}{l}\text { NIV } \\
\text { Complications } \\
\text { (n) }\end{array}$ & Limit \\
\hline $\begin{array}{l}\text { Padman et al. } \\
\text { (39) }\end{array}$ & $\begin{array}{l}\text { Monocenter } \\
\text { retrospective } \\
\text { study }\end{array}$ & $\begin{array}{l}11 \text { patients; } \\
\text { (range: 4-21 y) }\end{array}$ & $\begin{array}{l}\operatorname{DMD}(7), \text { SMA (2), } \\
\text { SCI (1), } \\
\text { nonspecific } \\
\text { myopathy (I) }\end{array}$ & Type 2 (11, 100\%) & $\begin{array}{l}\text { BLPAP via nasal } \\
\text { mask }\end{array}$ & $\begin{array}{l}\text { - NIV success rate (no intubation): } \\
91 \% \\
\text { - Improved RR, } \mathrm{PaCO}_{2} \text {, serum } \\
\text { bicarbonate, and length of } \\
\text { hospitalization after NIV use }\end{array}$ & $\begin{array}{l}\text { None } \\
\text { identified }\end{array}$ & $\begin{array}{l}\text { No major } \\
\text { complications }\end{array}$ & $\begin{array}{l}\text { Hypoxic ARF and } \\
\text { significant difficulty } \\
\text { handling } \\
\text { secretions }\end{array}$ \\
\hline $\begin{array}{l}\text { Birnkrant } \\
\text { et al. (55) }\end{array}$ & $\begin{array}{l}\text { Monocenter } \\
\text { retrospective } \\
\text { study }\end{array}$ & $\begin{array}{l}8 \text { patients (range } \\
1-18 y \text { ) }\end{array}$ & DMD(5), SMA(3) & $\begin{array}{l}\text { Undefined ARF, } \\
\text { including } 3 \\
\text { post-extubation ARF }\end{array}$ & $\begin{array}{l}\text { BLPAP via nasal } \\
\text { interface }\end{array}$ & $\begin{array}{l}\text { - Allowed weaning from an invasive } \\
\text { airway: } 100 \% \text { effective in avoiding } \\
\text { ETI or facilitating extubation }\end{array}$ & $\begin{array}{l}\text { None } \\
\text { identified }\end{array}$ & NA & Non described \\
\hline $\begin{array}{l}\text { Niranjan and } \\
\text { Bach (40) }\end{array}$ & $\begin{array}{l}\text { Monocenter } \\
\text { retrospective } \\
\text { study }\end{array}$ & $\begin{array}{l}10 \text { patients } \\
\text { (median: } 17 \mathrm{y} ; \\
\text { range: } 13-21 \mathrm{y} \text { ) vs. } \\
7 \text { historical } \\
\text { controls }\end{array}$ & $\begin{array}{l}\mathrm{DMD}(8), \mathrm{SMA}(1), \\
\mathrm{SCl}(1)\end{array}$ & $\begin{array}{l}\text { Type } 2(10,100 \%) \\
\text { including } 6 \\
\text { post-extubation ARF }\end{array}$ & $\begin{array}{l}\text { BLPAP via } \\
\text { mouthpiece or nasal } \\
\text { interface }+ \text { MI-E }\end{array}$ & $\begin{array}{l}\text { - NIV success rate (no intubation): } \\
100 \% \\
\text { - Shorter hospital stay in NIV group } \\
\text { than historical control }\end{array}$ & $\begin{array}{l}\text { None } \\
\text { identified }\end{array}$ & NA & Non described \\
\hline $\begin{array}{l}\text { Bach et al. } \\
\text { (56) }\end{array}$ & $\begin{array}{l}\text { Monocenter } \\
\text { retrospective } \\
\text { study }\end{array}$ & $\begin{array}{l}11 \text { children with } 28 \\
\text { ARF episodes } \\
\text { (median: } 6 \mathrm{~m} ; \\
\text { range: } 2-11 \mathrm{~m} \text { ) }\end{array}$ & SMA type 1 (11) & $\begin{array}{l}\text { Post-extubation ARF } \\
(28,100 \%)\end{array}$ & $\begin{array}{l}\text { BLPAP via nasal } \\
\text { interface+ MI-E for } \\
\text { post-extubation } \\
\text { support }\end{array}$ & $\begin{array}{l}\text { - NIV success rate (no intubation): } \\
82 \% \\
\text { - NIV can facilitate extubation for } \\
\text { type } 1 \text { SMA children even with } \\
\text { severe bulbar muscle weakness }\end{array}$ & $\begin{array}{l}\text { None } \\
\text { identified }\end{array}$ & NA & Non described \\
\hline $\begin{array}{l}\text { Vianello et al. } \\
\text { (36) }\end{array}$ & $\begin{array}{l}\text { Monocenter } \\
\text { prospective } \\
\text { case-control } \\
\text { study }\end{array}$ & $\begin{array}{l}14 \text { patients } \\
\text { (median: } 24 \text { y; } \\
\text { range: } 10-69 \text { y) vs. } \\
14 \text { historical } \\
\text { controls }\end{array}$ & $\begin{array}{l}\operatorname{DMD}(7), \operatorname{ALS}(4) \\
\mathrm{CMD}(1), \mathrm{HMSN} \\
(1), \mathrm{CM}(1)\end{array}$ & Type 2 (14, 100\%) & $\begin{array}{l}\mathrm{E}=\text { BLPAP via nasal } \\
\text { interface }+ \\
\text { cricothyroid-mini- } \\
\text { tracheostomy; } \mathrm{C}= \\
\text { IMV via ETI }\end{array}$ & $\begin{array}{l}\text { - NIV success rate (no intubation): } \\
71 \% \text { ( } 14 \% \text { mortality rate) vs. } 21 \% \\
\text { of controls ( } 57 \% \text { mortality rate) } \\
\text { - Lower mortality and } \\
\text { complications, and shorter ICU } \\
\text { stay of NIV group than controls } \\
\text { - NIV combined with } \\
\text { cricothyroid-mini-tracheostomy for } \\
\text { secretion clearance was well } \\
\text { tolerated without } \\
\text { significant complications }\end{array}$ & $\begin{array}{l}\text { None } \\
\text { identified }\end{array}$ & $\begin{array}{l}\text { No major } \\
\text { complications }\end{array}$ & $\begin{array}{l}\text { Severe bulbar } \\
\text { involvement }\end{array}$ \\
\hline $\begin{array}{l}\text { Vianello et al. } \\
\text { (37) }\end{array}$ & $\begin{array}{l}\text { Monocenter } \\
\text { prospective } \\
\text { case-control } \\
\text { study }\end{array}$ & $\begin{array}{l}11 \text { patients } \\
\text { (median: } 31 \mathrm{y} ; \\
\text { range: } 16-64 \text { y) vs. } \\
16 \text { historical } \\
\text { controls }\end{array}$ & $\begin{array}{l}\operatorname{DMD}(4), \operatorname{SMA}(3) \\
\operatorname{ALS}(2), \operatorname{LGMD}(1) \\
\operatorname{FSHD}(1)\end{array}$ & Type $2(11,100 \%)$ & $\begin{array}{l}\mathrm{E}=\mathrm{BLPAP} \text { via nasal } \\
\text { interface+ } \\
\mathrm{Ml}-\mathrm{E}+\mathrm{CPT} ; \mathrm{C}= \\
\mathrm{BLPAP}+\mathrm{CPT}\end{array}$ & $\begin{array}{l}\text { - NIV success rate (no intubation): } \\
82 \text { vs. } 37 \% \text { of controls } \\
\text { - No serious side effects and } \\
\text { well-tolerated in all subjects with } \\
\text { MI-E use }\end{array}$ & $\begin{array}{l}\text { None } \\
\text { identified }\end{array}$ & $\begin{array}{l}\text { Gastric } \\
\text { distension (1), } \\
\text { epistaxis (1) }\end{array}$ & \\
\hline $\begin{array}{l}\text { Servera et al. } \\
\text { (41) }\end{array}$ & $\begin{array}{l}\text { Monocenter } \\
\text { prospective } \\
\text { cohort study }\end{array}$ & $\begin{array}{l}17 \text { patients } \\
(48.7 \pm 20.9 \mathrm{y})\end{array}$ & $\begin{array}{l}\text { ALS (11), DMD (4), } \\
\text { transverse myelitis } \\
\text { (1), nonspecific } \\
\text { myopathy (1) }\end{array}$ & $\begin{array}{l}\text { Type } 2 \text { ARF (17, } \\
\text { 100\%) }\end{array}$ & $\begin{array}{l}\text { BLPAP via } \\
\text { nasal/oronasal } \\
\text { interfaces + MI-E }\end{array}$ & $\begin{array}{l}\text { - NIV success rate (no intubation): } \\
79.2 \% \\
\text { - Severe bulbar involvement limited } \\
\text { NIV effectiveness }\end{array}$ & $\begin{array}{l}\text { Bulbar } \\
\text { dysfunction }\end{array}$ & NA & $\begin{array}{l}\text { Severe bulbar } \\
\text { involvement } \\
\text { NIV/MI-E } \\
\text { performed in } \\
\text { non-ICU settings }\end{array}$ \\
\hline
\end{tabular}


TABLE 2 | Continued

\begin{tabular}{|c|c|c|c|c|c|c|c|c|c|}
\hline References & $\begin{array}{l}\text { Study } \\
\text { Design }\end{array}$ & $\begin{array}{l}\text { Number of NMD } \\
\text { patients (age) }\end{array}$ & $\begin{array}{l}\text { NMD diagnosis } \\
\text { (n) }\end{array}$ & ARF types* $(n, \%)$ & $\begin{array}{l}\text { NIV/interface/ } \\
\text { secretion } \\
\text { clearance }\end{array}$ & Success rate and main findings & $\begin{array}{l}\text { Predictor of } \\
\text { NIV failure }\end{array}$ & $\begin{array}{l}\text { NIV } \\
\text { Complications } \\
\text { (n) }\end{array}$ & Limit \\
\hline $\begin{array}{l}\text { Piastra et al. } \\
\text { (42) }\end{array}$ & $\begin{array}{l}\text { Monocenter } \\
\text { prospective } \\
\text { observational } \\
\text { cohort study }\end{array}$ & $\begin{array}{l}10 \text { children }(4.1 \pm \\
4.5 \mathrm{y} ; \text { range } 3 \\
\mathrm{~m}-12 \mathrm{y})\end{array}$ & $\begin{array}{l}\text { SMA type 1(2), } \\
\text { CMD -Ullrich (1), } \\
\text { CM-nemaline CM } \\
\text { (1), MG (2), } \\
\text { mitochondrial } \\
\text { myopathy (1), } \\
\text { spinal cord } \\
\text { hamartomatosis } \\
\text { (1), nonspecific } \\
\text { myopathies (2) }\end{array}$ & $\begin{array}{l}\text { Type } 2 \text { (5, 50\%); Type } \\
1 \text { (2, 20\%); } \\
\text { mixed/undefined ( } 3 \text {, } \\
30 \%)\end{array}$ & $\begin{array}{l}\text { BLPAP via facial } \\
\text { mask or helmet+ } \\
\text { CPT }\end{array}$ & $\begin{array}{l}\text { - NIV success rate (no intubation): } \\
80 \% \\
\text { - Hypercarbic ARF resolved within } \\
6 \mathrm{~h} \text { of NIV use } \\
\text { - Oxygenation markers improved } \\
\text { rapidly after NIV introduction }\end{array}$ & $\begin{array}{l}\text { Airway } \\
\text { obstruction }\end{array}$ & $\begin{array}{l}\text { No major } \\
\text { complications }\end{array}$ & $\begin{array}{l}\text { Copious tracheal } \\
\text { secretion needing } \\
\text { frequent suction }\end{array}$ \\
\hline $\begin{array}{l}\text { Dohna- } \\
\text { Schwake } \\
\text { et al. (53) }\end{array}$ & $\begin{array}{l}\text { Monocenter } \\
\text { retrospective } \\
\text { study }\end{array}$ & $\begin{array}{l}15 \text { children } \\
\text { (median: } 6 \text { y) }\end{array}$ & $\begin{array}{l}\text { SMA (6), DMD (3), } \\
\text { Pompe disease } \\
\text { (2); CMD (2), } \\
\text { myopathy (1), } \\
\text { myotonic } \\
\text { dystrophy (1) }\end{array}$ & $\begin{array}{l}\text { Undefined ARF, } \\
\text { including } 2 \\
\text { post-extubation ARF }\end{array}$ & CPAP via mask & $\begin{array}{l}\text { - NIV success rate (no intubation): } \\
87 \% \\
\text { - Improved } \mathrm{HR}, \mathrm{RR} \text {, blood } \mathrm{pH} \text {, } \\
\mathrm{PaCO}_{2} \text {, and } \mathrm{SaO}_{2} \text { after } 1-2 \mathrm{~h} \text { of } \\
\mathrm{NIV} \text { use in the success group }\end{array}$ & $\begin{array}{l}\text { Low } \mathrm{pH} \text { at } \\
1-2 \mathrm{~h} \text { after } \\
\mathrm{NIV}\end{array}$ & $\begin{array}{l}\text { midface skin } \\
\text { ulcers and } \\
\text { gastric } \\
\text { distension }\end{array}$ & $\begin{array}{l}3 \text { patients } \\
\text { requested } \\
\text { "do-not-intubate- } \\
\text { status" }\end{array}$ \\
\hline $\begin{array}{l}\text { Chen et al. } \\
(57)\end{array}$ & $\begin{array}{l}\text { Monocenter } \\
\text { prospective } \\
\text { observational } \\
\text { cohort study }\end{array}$ & $\begin{array}{l}15 \text { children with } 16 \\
\text { ARF episodes } \\
\text { (mean: } 8.1 \mathrm{y} \text {; } \\
\text { range } 3 \mathrm{~m}-18 \mathrm{y} \text { ) }\end{array}$ & $\begin{array}{l}\text { SMA (6), DMD (2), } \\
\text { CM (2), MM (2), } \\
\text { HMSN (2), LGMD } \\
21 \text { (1) }\end{array}$ & $\begin{array}{l}\text { Type } 2(15,94 \%) \\
\text { including } 1 \\
\text { post-extubation ARF; } \\
\text { Type } 1(1,6 \%)\end{array}$ & $\begin{array}{l}\text { BLPAP via } \\
\text { nasal/oronasal or } \\
\text { facial mask + MI-E }\end{array}$ & $\begin{array}{l}\text { - NIV success rate (no intubation): } \\
75 \% \\
\text { - Improved blood pH, and } \mathrm{PaCO}_{2} \\
\text { after } 12 \mathrm{~h} \text { of } \mathrm{NIV} \text { use in the } \\
\text { success group }\end{array}$ & $\begin{array}{l}\text { Fewer } \\
\text { decrement of } \\
\text { RR after } 3 \mathrm{~h} \\
\text { of NIV use }\end{array}$ & $\begin{array}{l}\text { No major } \\
\text { complications }\end{array}$ & \\
\hline $\begin{array}{l}\text { Chen et al. } \\
\text { (52) }\end{array}$ & $\begin{array}{l}\text { Monocenter } \\
\text { prospective } \\
\text { observational } \\
\text { cohort study }\end{array}$ & $\begin{array}{l}56 \text { NMD patients } \\
\text { ( } 44 \text { children) with } \\
62 \text { ARF episodes; } \\
\text { median: } 13 \text { y; } \\
\text { range: } 2 \text { m-39y) }\end{array}$ & $\begin{array}{l}\text { SMA (32), DMD } \\
\text { (14), CM (6), CMD } \\
\text { (4), MM (4), HMSN } \\
\text { (1), SMARD (1) }\end{array}$ & $\begin{array}{l}\text { Type } 2 \text { ARF (53, 85\%) } \\
\text { including } 23 \\
\text { post-extubation } \\
\text { failure; Type } 1 \text { ARF ( } 9 \text {, } \\
\text { 15\%) }\end{array}$ & $\begin{array}{l}\text { BLPAP via } \\
\text { nasal/oronasal or } \\
\text { facial mask + MI-E }\end{array}$ & $\begin{array}{l}\text { - NIV success rate (no intubation): } \\
86 \% \\
\text { - Improved HR, RR, blood pH, and } \\
\mathrm{PaCO}_{2} \text { after } 4 \mathrm{~h} \text { of NIV use in the } \\
\text { success group } \\
\text { - Shorter } \mathrm{PICU} \text { and hospital stay of } \\
\text { success group }\end{array}$ & $\begin{array}{l}\mathrm{RR} \\
\text { decreased at } \\
4 \mathrm{~h} ; \mathrm{pH} \\
\text { increased, } \\
\text { and } \mathrm{PaCO}_{2} \\
\text { decreased at } \\
\text { 4-8 h after } \\
\text { NIV }\end{array}$ & $\begin{array}{l}\text { No major } \\
\text { complications }\end{array}$ & $\begin{array}{l}\text { Initial checking } \\
\text { blood gases at a } \\
\text { later point of } 4-8 \mathrm{~h} \\
\text { after NIV }\end{array}$ \\
\hline
\end{tabular}

${ }^{*}$ Type 1 ARF, Hypoxemic ARF; Type 2 ARF, hypercapnic ARF.

NMD, neuromuscular disorders; NIV, non-invasive ventilation; ARF, acute respiratory failure; BLPAP, bi-level positive airway pressure; MI-E, Mechanical insufflator-exsufflator; E, experiment; C, control; CPT, chest physical treatments; DMD, Duchenne muscular dystrophy; SMA, spinal muscular atrophy; SCl, spinal cord injury, HMSN, hereditary motor and sensory neuropathy; CMD, congenital Muscular Dystrophy; CM, congenital Myopathy; MG, myasthenia gravis; MM, mitochondrial myopathy; SMARD, spinal muscular atrophy with respiratory distress; LGMD 21, limb-girdle muscular dystrophy type 21; NA, Not available.

CPAP, Continuous positive airway pressure. 
nasal interface (nasal cannula, nasal prong, or nasal mask) is recommended the interface of first choice $(6,44)$. Otherwise, choosing the right interface for older children is usually based on available materials and training of an experienced medical team, not on scientific data. Generally, in older children and young adults with ARF, full oronasal face masks are preferable to nasal interfaces because of better tolerance and a better sealing with less air leak $(60,62)$. Although some studies have shown that the feasibility and effectiveness of helmets in infants and young children, the experience of using helmets as interfaces in children is even rarer (62). It should be kept in mind that there is no single interface suitable for all situations, and the use of these interfaces in NMD children, especially in the critical care setting, requires better evidence support (59).

Recommendations for the initial setting of NIV are mainly based on clinical experience and expert consensus as there are no consistent data on optimal settings. If not contraindicated as the list aforementioned, the initial settings chosen should be disease and device-specific. Importantly, the information regarding the potential contraindications or complications related to NIV administration in the NMD patient population should be addressed $(6,11)$. Generally, the administration of NIV support should be set low initially to allow patient acclimation and then increase according to the physiologic needs and patient tolerance. According to our protocol specialized for NMD children, bilevel positive airway pressure (BLPAP) with an adequate interface is always effective in rescuing $\operatorname{ARF}(52,57)$. Especially during an acute chest infection, NIV should be used more intensively for these patients. Under adequate approaches of secretion clearance, supplemental oxygen may be added to NIV to maintain appropriate oxygenation. However, if the patient becomes almost whole-day dependent on NIV during an acute event, consider alternating masks to prevent pressure sores and alternate day and night between two ventilators of the same model so as not to run a ventilator continuously for days. The MI-E can be applied either in combination with NIV through a full-face mask or solitarily used in intubated patients via the endotracheal or tracheostomy tube with the cuff inflated. If applicable, supplementary manual augmentation of cough may be applied intermittently, followed by MI-E use.

In addition to noninvasive airway approaches, all other sensible standard measures can be taken during ARF episodes. These approaches include adapting a low threshold to deliver broad-spectrum antibiotics, adequate hydration, and attention to nutritional support. Humidification of the ventilator is often beneficial in reducing sputum viscosity and mobilizing secretions. Therapies of nebulized bronchodilator or systemic steroid may be considered if evidence of asthma or asthmatic bronchitis $(10,60)$.

From the perspective of chronic respiratory care, proactive use of NIV, and cough assistant MI-E in NMD children has been shown to reduce the rate of hospitalization and ICU admission (63-65). The familiarity of NMD patients with NIV use can help the effectiveness of NIV in the treatment of ARF (60). Several studies have shown that prior training of NIV and MI-E at home can contribute to a higher success rate in acute care settings $(14,52,66)$. In this regard, the proactive use of NIV and MI-E in the routine respiratory care of children with chronic NMD may also be beneficial (65).

Besides MI-E, high-frequency chest wall oscillation (HFCWO) has recently been proposed as a potential intervention used to facilitate secretion clearance in NMD patients. HFCWO delivers pressure to the chest wall accompanied by highfrequency vibration, which shows to move secretions from peripheral airways toward more central airways (67). However, the safety and effectiveness of HFCWO have not been well studied in managing ARF of NMD children, and its benefit in acute care settings is unclear (68). There is still a lack of data on the safety and effectiveness of NMD infants and young children known to be more susceptible to consistent and high frequent oscillation waves. Further research on HFCWO in NMD children is needed.

\section{CONTRAINDICATIONS AND COMPLICATIONS OF NIV AND MI-E}

The patient selection remains the most critical factor for the success of NIV in treating ARF. The contraindications to the NIV use include hemodynamic instability, severely decreased consciousness level, severe bulbar dysfunction (i.e., absence of gag reflex, or vocal cord paralysis), un-drained pneumothorax, facial deformity or injuries, recent surgery of facial, upper airway, or upper gastrointestinal tract, intolerance to NIV interface, multi-organ failure, life-threatening hypoxemia $\left(\mathrm{PaO}_{2}<60\right.$ mmHg with $\mathrm{FiO}_{2}>0.6$ ), and lack of familiarity of health-care provider with NIV operation $(6,14,62,69,70)$.

In general, NIV is a safe approach in managing ARF of infants and children with NMD, and the adverse effects described are minor (71). However, similar to any ventilation therapy, there are some adverse reactions and severe complications worthy of understanding. Reducing complications of NIV and MI-E largely depends on the well-trained and experienced staff of a multidisciplinary care team $(21,44,62,72)$. Gastric distension may occasionally occur, which can be ameliorated by nasogastric tube insertion and keeping adequate enteral feeding. Barotrauma may occur, but the risk is extremely low during NIV and much lower than during mechanical ventilation (73). For patients with hypovolemia, NIV should be used with caution, because NIV can cause an additional increase in intrathoracic pressure, which may result in a decrease in venous return (preload) and further deteriorate cardiac output (74).

Agitation may develop, especially during the initial interface placement on a child, but it is not necessary to discontinue NIV for this reason. Pharmacological sedation may be required, especially for children with NMD who receive NIV for the first time $(75,76)$. Choosing a more comfortable interface and finetuning NIV settings can reduce the need for sedatives (62). Other related complications include skin lesions, discomfort, claustrophobia, nasal mucosa trauma, and conjunctivitis, which may be prevented by a sophisticated selection of appropriate interface, alternating interface intermittently, and humidification of the ventilator $(59,60)$. 


\section{CONCLUSIONS}

The care of chronically progressive NMD has evolved significantly in the last decade, and many NMD children are now achieving prolonged survival through the advances in novel treatments (e.g., gene and molecular therapies) as well as respiratory care. However, there is still no consensus on the timing and limitations of NIV use in the treatment of ARF in children with NMD. Therefore, the administration protocol must be integrated with individualized clinical judgment. NMD usually includes various diseases of different severity, and the pathomechanism of ARF may vary with the type of NMD. Thus, it is unclear whether certain types of NMD may be more sensitive to NIV treatment for ARF. The variety and complexity of specific problems presented by different NMD necessitate separate remarks on the early recognition and adequate management of

\section{REFERENCES}

1. Aslan GK, Gurses HN, Issever H, Kiyan E. Effects of respiratory muscle training on pulmonary functions in patients with slowly progressive neuromuscular disease: a randomized controlled trial. Clin Rehabil. (2014) 28:573-81. doi: 10.1177/0269215513512215

2. Howard RS. Respiratory failure because of neuromuscular disease. Curr Opin Neurol. (2016) 29:592-601. doi: 10.1097/WCO.0000000000 000363

3. Boentert $M$, Wenninger $S$, Sansone VA. Respiratory involvement in neuromuscular disorders. Curr Opin Neurol. (2017) 30:52937. doi: $10.1097 /$ WCO. 0000000000000470

4. Hocker S. Primary acute neuromuscular respiratory failure. Neurol Clin. (2017) 35:707-21. doi: 10.1016/j.ncl.2017.06.007

5. Yuan N. Neuromuscular disease and the pulmonologist. Curr Opin Pediatr. (2012) 24:336-43. doi: 10.1097/MOP.0b013e3283531bb0

6. Panitch HB. Respiratory implications of pediatric neuromuscular disease. Respir Care. (2017) 62:826-48. doi: 10.4187/respcare.05250

7. Benditt JO, Boitano LJ. Pulmonary issues in patients with chronic neuromuscular disease. Am J Respir Crit Care Med. (2013) 187:104655. doi: $10.1164 / \mathrm{rccm} .201210-1804 \mathrm{CI}$

8. Mehta S. Neuromuscular disease causing acute respiratory failure. Respir Care. (2006) 51:1016-21; discussion 21-3.

9. Panitch HB. The pathophysiology of respiratory impairment in pediatric neuromuscular diseases. Pediatrics. (2009) 123 Suppl 4:S215-8. doi: 10.1542/peds.2008-2952C

10. Voulgaris A, Antoniadou M, Agrafiotis M, Steiropoulos P. Respiratory involvement in patients with neuromuscular diseases: a Narrative review. Pulm Med. (2019) 2019:2734054. doi: 10.1155/2019/2734054

11. Hull J, Aniapravan R, Chan E, Chatwin M, Forton J, Gallagher J, et al. British thoracic society guideline for respiratory management of children with neuromuscular weakness. Thorax. (2012) 67 Suppl 1:i140. doi: 10.1136/thoraxjnl-2012-201964

12. Shahrizaila N, Kinnear WJ, Wills AJ. Respiratory involvement in inherited primary muscle conditions. J Neurol Neurosurg Psychiatry. (2006) 77:110815. doi: 10.1136/jnnp.2005.078881

13. Rezania K, Goldenberg FD, White S. Neuromuscular disorders and acute respiratory failure: diagnosis and management. Neurol Clin. (2012) 30:161-85, viii. doi: 10.1016/j.ncl.2011.09.010

14. Racca F, Del Sorbo L, Mongini T, Vianello A, Ranieri VM. Respiratory management of acute respiratory failure in neuromuscular diseases. Minerva Anestesiol. (2010) 76:51-62.

15. Hill NS. Neuromuscular disease in respiratory and critical care medicine. Respir Care. (2006) 51:1065-71.

16. Khirani S, Bersanini C, Aubertin G, Bachy M, Vialle R, Fauroux B. Noninvasive positive pressure ventilation to facilitate the post-operative
ARF in children with NMD. More future researches designed specifically for the pediatric NMD population are still needed, and several issues remain to be clarified.

\section{AUTHOR CONTRIBUTIONS}

T-HC and J-HH contributed to conception and design, acquisition of data, revising the manuscript critically for relevant intellectual content, and final approval of the version to be published. All authors read and approved the final manuscript.

\section{FUNDING}

This study was funded by a research grant from Kaohsiung Medical University Hospital, Kaohsiung, Taiwan (KMUH-108-8R47). respiratory outcome of spine surgery in neuromuscular children. Eur Spine J. (2014) 23 Suppl 4:S406-11. doi: 10.1007/s00586-014-3335-6

17. Bach JR, Goncalves MR, Hon A, Ishikawa Y, De Vito EL, Prado F, et al. Changing trends in the management of end-stage neuromuscular respiratory muscle failure: recommendations of an international consensus. Am J Phys Med Rehabil. (2013) 92:267-77. doi: 10.1097/PHM.0b013e31826edcf1

18. Wang CH, Bonnemann CG, Rutkowski A, Sejersen T, Bellini J, Battista V, et al. Consensus statement on standard of care for congenital muscular dystrophies. J Child Neurol. (2010) 25:1559-81. doi: 10.1177/0883073810381924

19. Wang CH, Dowling JJ, North K, Schroth MK, Sejersen T, Shapiro F, et al. Consensus statement on standard of care for congenital myopathies. J Child Neurol. (2012) 27:363-82. doi: 10.1177/0883073812436605

20. Wang CH, Finkel RS, Bertini ES, Schroth M, Simonds A, Wong B, et al. Consensus statement for standard of care in spinal muscular atrophy. J Child Neurol. (2007) 22:1027-49. doi: 10.1177/0883073807305788

21. Finkel RS, Mercuri E, Meyer OH, Simonds AK, Schroth MK, Graham $\mathrm{RJ}$, et al. Diagnosis and management of spinal muscular atrophy: part 2: pulmonary and acute care; medications, supplements and immunizations; other organ systems; and ethics. Neuromuscul Disord. (2018) 28:197207. doi: $10.1016 /$ j.nmd.2017.11.004

22. Schroth MK. Special considerations in the respiratory management of spinal muscular atrophy. Pediatrics. (2009) 123 Suppl 4:S2459. doi: 10.1542/peds.2008-2952K

23. Bushby K, Finkel R, Birnkrant DJ, Case LE, Clemens PR, Cripe L, et al. Diagnosis and management of duchenne muscular dystrophy, part 2: implementation of multidisciplinary care. Lancet Neurol. (2010) 9:17789. doi: 10.1016/S1474-4422(09)70272-8

24. Poponick JM, Jacobs I, Supinski G, DiMarco AF. Effect of upper respiratory tract infection in patients with neuromuscular disease. Am J Respir Crit Care Med. (1997) 156(2 Pt 1):659-64. doi: 10.1164/ajrccm.156.2.9611029

25. Katz SL, Gaboury I, Keilty K, Banwell B, Vajsar J, Anderson $\mathrm{P}$, et al. Nocturnal hypoventilation: predictors and outcomes in childhood progressive neuromuscular disease. Arch Dis Child. (2010) 95:998-1003. doi: 10.1136/adc.2010.182709

26. Yates K, Festa M, Gillis J, Waters K, North K. Outcome of children with neuromuscular disease admitted to paediatric intensive care. Arch Dis Child. (2004) 89:170-5. doi: 10.1136/adc.2002.019562

27. Cabrera Serrano M, Rabinstein AA. Causes and outcomes of acute neuromuscular respiratory failure. Arch Neurol. (2010) 67:1089-94. doi: 10.1001/archneurol.2010.207

28. Vianello A, Arcaro G, Braccioni F, Gallan F, Marchi MR, Chizio S, et al. Prevention of extubation failure in high-risk patients with neuromuscular disease. J Crit Care. (2011) 26:517-24. doi: 10.1016/j.jcrc.2010.12.008

29. Katz S, Selvadurai H, Keilty K, Mitchell M, MacLusky I. Outcome of noninvasive positive pressure ventilation in paediatric neuromuscular disease. Arch Dis Child. (2004) 89:121-4. doi: 10.1136/adc.2002.018655 
30. Mercuri E, Finkel RS, Muntoni F, Wirth B, Montes J, Main M, et al. Diagnosis and management of spinal muscular atrophy: part 1: recommendations for diagnosis, rehabilitation, orthopedic and nutritional care. Neuromuscul Disord. (2018) 28:103-15. doi: 10.1016/j.nmd.2017.11.005

31. Yanez LJ, Yunge M, Emilfork M, Lapadula M, Alcantara A, Fernandez C, et al. A prospective, randomized, controlled trial of noninvasive ventilation in pediatric acute respiratory failure. Pediatr Crit Care Med. (2008) 9:4849. doi: 10.1097/PCC.0b013e318184989f

32. Najaf-Zadeh A, Leclerc F. Noninvasive positive pressure ventilation for acute respiratory failure in children: a concise review. Ann Intensive Care. (2011) 1:15. doi: 10.1186/2110-5820-1-15

33. Stauffer JL, Olson DE, Petty TL. Complications and consequences of endotracheal intubation and tracheotomy. A prospective study of 150 critically ill adult patients. Am J Med. (1981) 70:65-76. doi: 10.1016/0002-9343(81)90413-7

34. Konrad F, Schreiber T, Brecht-Kraus D, Georgieff M. Mucociliary transport in iCU patients. Chest. (1994) 105:237-41. doi: 10.1378/chest.105.1.237

35. Rivera R, Tibballs J. Complications of endotracheal intubation and mechanical ventilation in infants and children. Crit Care Med. (1992) 20:1939. doi: 10.1097/00003246-199202000-00008

36. Vianello A, Bevilacqua M, Arcaro G, Gallan F, Serra E. Noninvasive ventilatory approach to treatment of acute respiratory failure in neuromuscular disorders. A comparison with endotracheal intubation. Intensive Care Med. (2000) 26:384-90. doi: 10.1007/s001340051171

37. Vianello A, Corrado A, Arcaro G, Gallan F, Ori C, Minuzzo M, et al. Mechanical insufflation-exsufflation improves outcomes for neuromuscular disease patients with respiratory tract infections. Am J Phys Med Rehabil. (2005) 84:83-8; discussion 9-91. doi: 10.1097/01.PHM.0000151941.97266.96

38. Rabinstein A, Wijdicks EF. BiPAP in acute respiratory failure due to myasthenic crisis may prevent intubation. Neurology. (2002) 59:16479. doi: 10.1212/01.WNL.0000033797.79530.16

39. Padman R, Lawless S, Von Nessen S. Use of BiPAP by nasal mask in the treatment of respiratory insufficiency in pediatric patients: preliminary investigation. Pediatr Pulmonol. (1994) 17:119-23. doi: 10.1002/ppul.1950170208

40. Niranjan V, Bach JR. Noninvasive management of pediatric neuromuscular ventilatory failure. Crit Care Med. (1998) 26:2061-5. doi: 10.1097/00003246-199812000-00042

41. Servera E, Sancho J, Zafra MJ, Catala A, Vergara P, Marin J. Alternatives to endotracheal intubation for patients with neuromuscular diseases. Am J Phys Med Rehabil. (2005) 84:851-7. doi: 10.1097/01.phm.0000184097.17189.93

42. Piastra M, Antonelli M, Caresta E, Chiaretti A, Polidori G, Conti G. Noninvasive ventilation in childhood acute neuromuscular respiratory failure: a pilot study. Respiration. (2006) 73:791-8. doi: 10.1159/000090777

43. Panitch HB. Airway clearance in children with neuromuscular weakness. Curr Opin Pediatr. (2006) 18:277-81. doi: 10.1097/01.mop.0000193297.40388.19

44. Demaret P, Mulder A, Loeckx I, Trippaerts M, Lebrun F. Noninvasive ventilation is useful in paediatric intensive care units if children are appropriately selected and carefully monitored. Acta Paediatr. (2015) 104:861-71. doi: 10.1111/apa.13057

45. Chatwin M, Toussaint M, Goncalves MR, Sheers N, Mellies U, Gonzales-Bermejo J, et al. Airway clearance techniques in neuromuscular disorders: a state of the art review. Respir Med. (2018) 136:98-110. doi: 10.1016/j.rmed.2018.01.012

46. Tzeng AC, Bach JR. Prevention of pulmonary morbidity for patients with neuromuscular disease. Chest. (2000) 118:13906. doi: 10.1378/chest.118.5.1390

47. Rabinstein AA. Noninvasive ventilation for neuromuscular respiratory failure: when to use and when to avoid. Curr Opin Crit Care. (2016) 22:949. doi: 10.1097/MCC.0000000000000284

48. Panitch HB. Respiratory issues in the management of children with neuromuscular disease. Respir Care. (2006) 51:885-93; discussion 94-5.

49. Auger C, Hernando V, Galmiche H. Use of mechanical insufflationExsufflation devices for airway clearance in subjects with neuromuscular disease. Respir Care. (2017) 62:236-45. doi: 10.4187/respcare.04877

50. Morrow B, Zampoli M, van Aswegen H, Argent A. Mechanical insufflationexsufflation for people with neuromuscular disorders. Cochrane Database Syst Rev. (2013) 12:CD010044. doi: 10.1002/14651858.CD010044.pub2
51. Mustfa N, Aiello M, Lyall RA, Nikoletou D, Olivieri D, Leigh PN, et al. Cough augmentation in amyotrophic lateral sclerosis. Neurology. (2003) 61:12857. doi: 10.1212/01.WNL.0000092018.56823.02

52. Chen TH, Liang WC, Chen IC, Liu YC, Hsu JH, Jong YJ. Combined noninvasive ventilation and mechanical insufflator-exsufflator for acute respiratory failure in patients with neuromuscular disease: effectiveness and outcome predictors. Ther Adv Respir Dis. (2019) 13:28. doi: 10.1177/1753466619875928

53. Dohna-Schwake C, Stehling F, Tschiedel E, Wallot M, Mellies U. Noninvasive ventilation on a pediatric intensive care unit: feasibility, efficacy, and predictors of success. Pediatr Pulmonol. (2011) 46:111420. doi: 10.1002/ppul.21482

54. Bach JR, Goncalves MR, Hamdani I, Winck JC. Extubation of patients with neuromuscular weakness: a new management paradigm. Chest. (2010) 137:1033-9. doi: 10.1378/chest.09-2144

55. Birnkrant DJ, Pope JF, Eiben RM. Pediatric noninvasive nasal ventilation. J Child Neurol. (1997) 12:231-6. doi: 10.1177/088307389701200401

56. Bach JR, Niranjan V, Weaver B. Spinal muscular atrophy type 1: a noninvasive respiratory management approach. Chest. (2000) 117:11005. doi: 10.1378/chest.117.4.1100

57. Chen TH, Hsu JH, Wu JR, Dai ZK, Chen IC, Liang WC, et al. Combined noninvasive ventilation and mechanical in-exsufflator in the treatment of pediatric acute neuromuscular respiratory failure. Pediatr Pulmonol. (2014) 49:589-96. doi: 10.1002/ppul.22827

58. Keenan SP, Sinuff T, Burns KE, Muscedere J, Kutsogiannis J, Mehta S, et al. Clinical practice guidelines for the use of noninvasive positive-pressure ventilation and noninvasive continuous positive airway pressure in the acute care setting. CMAJ. (2011) 183:E195-214. doi: 10.1503/cmaj.100071

59. Pavone M, Verrillo E, Caldarelli V, Ullmann N, Cutrera R. Noninvasive positive pressure ventilation in children. Early Hum Dev. (2013) 89 Suppl 3:S25-31. doi: 10.1016/j.earlhumdev.2013.07.019

60. Simonds AK. Ventilator support in children with neuromuscular disorders. In: Sterni LM, Carroll JL, editors. Caring for the Ventilator Dependent Child: A Clinical Guide. New York, NY: Springer. (2016). p. 28398. doi: 10.1007/978-1-4939-3749-3_14

61. Fauroux B, Lavis JF, Nicot F, Picard A, Boelle PY, Clement A, et al. Facial side effects during noninvasive positive pressure ventilation in children. Intensive Care Med. (2005) 31:965-9. doi: 10.1007/s00134-005-2669-2

62. Viscusi CD, Pacheco GS. Pediatric emergency noninvasive ventilation. Emerg Med Clin North Am. (2018) 36:387-400. doi: 10.1016/j.emc.2017. 12.007

63. Young HK, Lowe A, Fitzgerald DA, Seton C, Waters KA, Kenny E, et al. Outcome of noninvasive ventilation in children with neuromuscular disease. Neurology. (2007) 68:198-201. doi: 10.1212/01.wnl.0000251299.54608.13

64. Simonds AK. Respiratory support for the severely handicapped child with neuromuscular disease: ethics and practicality. Semin Respir Crit Care Med. (2007) 28:342-54. doi: 10.1055/s-2007-981655

65. Lemoine TJ, Swoboda KJ, Bratton SL, Holubkov R, Mundorff M, Srivastava R. Spinal muscular atrophy type 1: are proactive respiratory interventions associated with longer survival? Pediatr Crit Care Med. (2012) 13:e1615. doi: 10.1097/PCC.0b013e3182388ad1

66. Sancho J, Servera E, Diaz J, Marin J. Efficacy of mechanical insufflationexsufflation in medically stable patients with amyotrophic lateral sclerosis. Chest. (2004) 125:1400-5. doi: 10.1378/chest.125.4.1400

67. Lechtzin N, Wolfe LF, Frick KD. The impact of high-Frequency chest wall oscillation on healthcare use in patients with neuromuscular diseases. Ann Am Thorac Soc. (2016) 13:904-9. doi: 10.1513/AnnalsATS.201509-597OC

68. Yuan N, Kane P, Shelton K, Matel J, Becker BC, Moss RB. Safety, tolerability, and efficacy of high-frequency chest wall oscillation in pediatric patients with cerebral palsy and neuromuscular diseases: an exploratory randomized controlled trial. J Child Neurol. (2010) 25:81521. doi: $10.1177 / 0883073809350223$

69. Racca F, Vianello A, Mongini T, Ruggeri P, Versaci A, Vita GL, et al. Practical approach to respiratory emergencies in neurological diseases. Neurol Sci. (2020) 41:497-508. doi: 10.1007/s10072-019-04163-0

70. Chen TH, Hsu JH, Jong YJ. Noninvasive airway approaches for acute neuromuscular respiratory failure in emergency departments. Pediatr Pulmonol. (2017) 52:E55-E7. doi: 10.1002/ppul.23693 
71. Calderini E, Chidini G, Pelosi P. What are the current indications for noninvasive ventilation in children? Curr Opin Anaesthesiol. (2010) 23:368-74. doi: 10.1097/ACO.0b013e3283 39507b

72. Gay PC. Complications of noninvasive ventilation in acute care. Respir Care. (2009) 54:246-57; discussion 57-8.

73. Modi HN, Suh SW, Hong JY, Cho JW, Park JH, Yang JH. Treatment and complications in flaccid neuromuscular scoliosis (Duchenne muscular dystrophy and spinal muscular atrophy) with posterior-only pedicle screw instrumentation. Eur Spine J. (2010) 19:384-93. doi: 10.1007/s00586-0091198-z

74. Carron M, Freo U, BaHammam AS, Dellweg D, Guarracino F, Cosentini R, et al. Complications of noninvasive ventilation techniques: a comprehensive qualitative review of randomized trials. Br J Anaesth. (2013) 110:896914. doi: 10.1093/bja/aet070

75. Abadesso C, Nunes P, Silvestre C, Matias E, Loureiro H, Almeida H. Noninvasive ventilation in acute respiratory failure in children. Pediatr Rep. (2012) 4:e16. doi: 10.4081/pr 2012.e16

76. Wolfler A, Calderini E, Iannella E, Conti G, Biban P, Dolcini A, et al. Evolution of noninvasive mechanical ventilation use: a Cohort study among Italian PICUs. Pediatr Crit Care Med. (2015) 16:41827. doi: 10.1097/PCC.0000000000000387

Conflict of Interest: The authors declare that the research was conducted in the absence of any commercial or financial relationships that could be construed as a potential conflict of interest.

Copyright (C) 2020 Chen and Hsu. This is an open-access article distributed under the terms of the Creative Commons Attribution License (CC BY). The use, distribution or reproduction in other forums is permitted, provided the original author(s) and the copyright owner(s) are credited and that the original publication in this journal is cited, in accordance with accepted academic practice. No use, distribution or reproduction is permitted which does not comply with these terms. 